\title{
The Prospect of Genotyping Assay-Point of Care (Individualized Medicine): The Fast Track
}

\author{
Asim Ahmed Elnour ${ }^{1 *}$, Abdulla Shehab ${ }^{2}$, Akshaya Srikanth Bhagavathula ${ }^{3}$, Abdulla A A Al \\ Amoodi $^{2}$, Omer Abdulla Shehab ${ }^{2}$, Saif Al Naimi ${ }^{4}$, Naama MS Al Kalbani ${ }^{4}$, Alaa Abdul Aziz ${ }^{1}$, \\ Mahmoud Yasser Eldeeb ${ }^{5}$, Mirai Mourad Sadek ${ }^{6}$ and Pinar Erkekoglu ${ }^{7}$ \\ ${ }^{1}$ Pharmacology Department, College of Medicine and Health Sciences, UAE University, and; Resident Program of Internal Medicine-Tawam Hospital, \\ Abu Dhabi Health Services-SEHA, UAE \\ ${ }^{2}$ Internal Medicine Department, College of Medicine and Health Sciences (CMHS), UAE University, UAE \\ ${ }^{3}$ Department of Clinical Pharmacy, University of Gondar College of Medicine and Health Sciences, Gondar, Ethiopia \\ ${ }^{4}$ Tawam Hospital, Abu Dhabi Health Services-SEHA, UAE \\ ${ }^{5}$ Internal Medicine Dept, Resident Year 1, Academic Affairs, Al-Ain Hospital, UAE \\ ${ }^{6}$ Pharmacist, Madinat Mohammed Bin Zayed Primary Health Care Center, Ambulatory Health Services, Abu Dhabi Health Services (SEHA), UAE \\ ${ }^{7}$ Associate Professor, Department of Toxicology, Faculty of Pharmacy, Hacettepe University, Sihhiye 06100, Ankara, Turkey
}

Received: February 18, 2015, Accepted: April 27, 2015, Published: May 07, 2015

*Corresponding author: Asim Ahmed Elnour, Associate Professor, Pharmacology Department, College of Medicine and Health Sciences, UAE University, Al Ain, UAE, Tel: +971-506-231-502; E-mail: assahura1962@uaeu.ac.ae

\begin{abstract}
Background: The basis of the genotyping assay is derived from the worldwide data which suggested that the pharmacologic effects of Clopidogrel vary based on CYP2C19 genotype. However; it is not apparent if this interprets into adverse clinical outcomes. It is imperative to examine the risk of adverse cardiovascular outcomes in patients treated with Clopidogrel who carry 1 or 2 reduced-function CYP2C19 genetic variants in other population that preliminary data from the western countries suggests is more frequent.
\end{abstract}

Aim: The aim of this short communication is to raise the awareness of the presence of alleles CYP4502C19*2 in some populations receiving Clopidogrel. We need to investigate the existence of CYP2C19 genetic variants in some populations such as African, Asians (Chinese) and Middle East (Arabs). The risk of major adverse cardiovascular outcomes in patients treated with Clopidogrel who carries any form of CYP2C19 genetic variants needs further attention. We need to explore the use of genotyping assay as an evolving approach to individualized medicine.

The Proposed Research: Patients are in great need for this sophisticated technology coupled with experience derived by researches that delineate the Clopidogrel-genetic variability of certain populations. It has been well demonstrated that detecting a patient's polymorphism is essential to understand his or her clinical response. Genetic testing can in assisting with allele determination, and pairing this result to a specification of a patient's level of drug metabolism response (extensive, intermediate, and poor). Testing, independent of prior or concurrent usage of Clopidogrel, reveals whether a patient should be considered for Clopidogrel or alternative (Prasugrel or Ticagrelor).

Keywords: Clopidogrel Allele; Genotyping Assay; Point of care; Spartan RX

\section{Introduction}

The platelet aggregation plays a fundamental and important role in the pathogenesis of arterial thrombosis in Coronary Heart Diseases-CHD [1,2], stroke and Peripheral Arterial Disease-PAD [3]. However, the contribution of genetic influences on interindividual variation in platelet aggregability is not characterized in certain population such as that in the Middle East. Despite great progress in diagnosis and treatment of coronary syndromes worldwide, the estimated absolute risk of recurring vascular events amongst patients taking platelet inhibitors remains relatively elevated.

Clopidogrel, a proven anti-platelet therapy, is a pro-drug activated in the liver to its potent form by the Cytochrome P450 (CYP450) CYP4502C19 Cytochrome system. Polymorphisms (genetic variants) in the CYP2C19 gene $(* 2, * 3, * 4, * 5$ and *17) confer reduced enzyme function, engendering less hepatic alteration of Clopidogrel to its active metabolite, and less subsequent blockage of platelet ADP sub-receptors to prevent platelet aggregation. Such reduction may be compounded by already-reduced amounts of available pro-drug substrate - up to eighty five percent of inactive Clopidogrel is degraded from the outset by intestinal esterases.

Genetic variance is seen in approximately 30\% of whites, $40 \%$ of blacks, and more than $55 \%$ of East Asians [4]. Undeniably, polymorphisms are noteworthy, as they significantly reduce the response to Clopidogrel by approximately one quarter to one third [4]. Clearly, the heterogeneity in patient response to Clopidogrel, based on individual genetics, can greatly affect clinical response.

Effective medical treatment following Acute Coronary Syndromes (ACS) and Percutaneous Coronary Intervention 
(PCI) consists of dual anti-platelet therapy with aspirin and Clopidogrel. Despite this treatment approach, a considerable portion of patients continue to experience an amplified rate of subsequent adverse Cardio Vascular Events (CVEs) including death, fatal/non fatal Myocardial Infarction (MI), and stent thrombosis. This unrelenting vulnerability has been documented to be associated with inadequate platelet inhibition in response to Clopidogrel administration, an observable fact referred to as Clopidogrel resistance.

\section{The Phenomenon of Clopidogrel Resistance}

Although multiple factors have been implicated in Clopidogrel resistance, mounting evidence suggests a crucial role for the loss-of-function of CYP2C19*2 genetic variant, [5]. Presence of the *2 allele has been associated with a 1.5- to 6-fold increased risk of cardiovascular death, MI (fatal and non-fatal), and stent thrombosis following PCI in patients treated with Clopidogrel. These findings, recently reinforced by a meta-analysis, led the American Food and Drug Administration (FDA) to issue a boxed warning for Clopidogrel that poor metabolizers may not receive the full protective benefit of the drug.

Consequently, experts have recommended routine genotyping in the context of dual anti-platelet therapy following PCI. A personalized approach to dual anti-platelet therapy following PCI is feasible given the treatment options such as Prasugrel (Effien $t^{\circledR}$ ) and Ticagrelor (Brilinta ${ }^{\circledR}$ ) which are possible candidates for overcoming Clopidogrel resistance but may warrant head to head trial regarding the comparative and long term efficacy.

Selective administration of Prasugrel to patients at increased risk of Clopidogrel resistance has potential to minimize adverse ischemic events, while simultaneously minimizing associated bleeding events and costs.

There were two studies in 2009, which were published in New England Journal of Medicine that authenticate this fact. The first study has concluded that patients treated with Clopidogrel, "carriers of a reduced-function CYP2C19 allele had significantly lower levels of the active metabolite of Clopidogrel, diminished platelet inhibition, and a higher rate of Major Adverse Cardiovascular Events (MACE), than did non-carriers of the alleles" [4]. The second study revealed that patients with an acute MI who were receiving Clopidogrel, "those carrying CYP2C19 loss-of-function alleles had a higher rate of subsequent CVEs than those of their counterparts" [6]. Meta-analysis of 9 studies has shown that patients with reduced-function CYP2C19 alleles treated with Clopidogrel are more likely to experience cardiovascular death, MI, or stroke than patients who were not carriers of reduced-function alleles $[7,8]$ and had a significantly increased risk of stent thrombosis $[9,10]$.

\section{Significance of the Problem}

The significance of the problem is to reveal the percent of population exhibiting CYP4502C19 reduced activity (prevalence) and the distributions of variant alleles across certain population such as the Middle East in Clopidogrel users. The need to identify the clinical significance of CYP4502C19 reduced activity on patients' clinical outcomes.

Also the feasibility of point-of-care genotyping and incidence of stent thrombosis are of great importance. We need to research the genotyping assay for improved cardiovascular outcomes to improve individualized-medicine benefits - based on evidence data and the need to explore the phamcoeconomic impact of genotyping on patient outcomes.

\section{Clopidogrel Genotyping Assay}

A prospective pharmacogenomic approach to anti-platelet therapy has been previously susceptible to limited access and the time-impediment associated with genetic testing (may take 2 to 5 days). The advanced technology made of a point-of-care CYP2C19*2 genetic tests that require least training to operate overcome these limitations, and may facilitate the incorporation of pharmacogenomic strategies into routine current anti-platelet therapy in clinical practice.

\section{The kit to be used}

Spartan RX platform for CYP2C19 for therapeutics that are metabolized by P450 2C19and are specifically affected by $* 2, * 3, * 17$ alleles. Spartan RX from Spartan Bioscience Inc. instrument is fast and accurate for DNA analysis in point-of-care applications. The detailed patient result, which will be one of the following:

CYP2C19*1/*1 - The patient does not carry any CYP2C19*2 allele

- CYP2C19*2/*1 - The patient carries one CYP2C19*2 allele

- CYP2C19*2/*2 - The patient carries two CYP2C19*2 alleles

- Inconclusive - The patient test needs to be repeated

This validated instrument is equipped with software notebook and printer. The sample obtained from buccal swab enable results to be printed in an hour.

\section{Summary}

The evidence supporting the genotyping of Clopidogrel continues with more studies in the horizons from across the globe and from populations in the Middle East such as from Saudi Arabia and United Arab Emirates. The importance of these ongoing studies will rely on their outcomes regarding the presence of alleles that poses the Clopidogrel resistance. The new emerging genotyping assay has moved fast over the last few years and the pharmacists have joined the race.

Recently, in Canada the community pharmacists has commenced genotyping assay with the one hour device and continues to play a role in improving the clinical outcomes of patients eligible for anti-platelet Clopidogrel. In the very near future we are going to witness more drugs to join the track for genotyping assay such as antipsychotics (with polymorphism). Many firms in United States has provided pharmacogenetic tests 
for research or clinical trials for a number of enzymes responsible for candidates drugs such as Cytochrome P450 2D6, P450 2C9, VKORC1, P450 3A4/3A5, P450 1A2,N-Acetyl Transferase 2 (NAT2) Genotyping.

Information concerning genetic testing (CYP4502C19 alleles), the role of extended genotypic classification, dose adjustment, and the effect of customized therapeutic assortment on thrombotic and hemorrhagic outcomes across a wide spectrum of patients (populations) deserve further attention and research.

\section{References}

1. Fuster V, Badimon L, Badimon JJ, Chesebro JH. The pathogenesis of coronary artery disease and the acute coronary syndromes, N Engl Med. 1992; 326(5): 310-8.

2. Fitzgerald DJ, Roy L, Catella F, FitzGerald GA. Platelet activation in unstable coronary disease. N Engl J Med. 1986; 315(16): 983-9.

3. del Zoppo GJ, Hallenbeck JM. Advances in the vascular pathophysiology of ischemic stroke. Thromb Res. 2000; 98(3): 73-81.

4. Mega JL, Close SL, Wiviott SD, Shen L, Hockett RD, Brandt JT, et al. Cytochrome P-450 Polymorphisms and Response to Clopidogrel. N Engl J Med. 2009; 360(4): 354-62. doi: 10.1056/NEJMoa0809171.

5. Scott SA, Sangkuhl K, Gardner EE, Stein CM, Hulot JS, Johnson JA, et al. Clinical Pharmacogenetics Implementation Consortium guidelines for cytochrome P450-2C19 (CYP2C19) genotype and clopidogrel therapy. Clin Pharmacol Ther. 2011; 90(2): 328-32. doi: 10.1038/ clpt.2011.132.

6. Simon T, Verstuyft C, Mary-Krause M, Quteineh L, Drouet E, Méneveau N, et al. Genetic determinants of response to clopidogrel and cardiovascular events. N Engl J Med. 2009; 360(4): 363-75. doi: 10.1056/NEJMoa0808227.

7. Mega JL, Simon T, Collet JP, Anderson JL, Antman EM, Bliden K, et al. Reduced-function CYP2C19 genotype and risk of adverse clinical outcomes among patients treated with clopidogrel predominantly for PCI: a meta-analysis. JAMA. 2010; 304(16):1821-30. doi: 10.1001/ jama.2010.1543.

8. Hulot JS, Collet JP, Silvain J, Pena A, Bellemain-Appaix A, Barthélémy 0 , et al. Cardiovascular risk in clopidogrel-treated patients according to cytochrome P450 2C19*2 loss-of-function allele or proton pump inhibitor coadministration: a systematic meta-analysis. J Am CollCardiol. 2010; 56(2): 134-43. doi: 10.1016/j.jacc.2009.12.071.

9. Reassessment of anti-platelet therapy using an individualized strategy based on genetic evaluation RAPID Gene. Not published yet. Announced results. Nov. 2011.

10. Fontana P, Cattaneo M, Combescure C, Reny JL. Tailored Thienopyridine Therapy: No Urgency for CYP2C19 Genotyping. J Am Heart Assoc. 2013; 2(2): e000131. doi: 10.1161/JAHA.112.000131. 